\title{
Bentham Obras: Um Sistema de Gestão Inteligente para a Construção Civil
}

\author{
Iesus Batista \\ iesus90@gmail.com \\ Instituto Federal do Ceará \\ Renato Oliveira \\ renatoalves97.123@gmail.com \\ Instituto Federal do Ceará
}

\author{
Alice Feitosa \\ alice30feitosabarbosa@gmail.com \\ Instituto Federal do Ceará \\ Matheus Gurgel \\ mateusgiqui@hotmail.com \\ Instituto Federal do Ceará
}

\author{
Luan Douglas \\ douglasmaia.mn.sl@gmail.com \\ Instituto Federal do Ceará \\ Arthur Bezerra \\ arthurbezerra92@gmail.com \\ Quanta Consultoria
}

\begin{abstract}
Carina Oliveira
carina.oliveira@ifce.edu.br

Instituto Federal do Ceará
\end{abstract}

\begin{abstract}
Nowadays, construction management is the main challenge for civil engineering corporations mainly due to the large number of information collected daily. In parallel, the adoption of paper based resources in the management process increases the chances of obtaining inefficient results. Besides that, it is important to care about environmental aspects, where each construction must be less harmful to nature. In this context, this paper presents Bentham Obras, a system based on Web and Mobile platforms that provides several management processes, such as financial monitoring, work schedule, task execution control and contract accomplishment. After using the system for a period of 8 months, civil engineering corporations that have adopted the system highlighted the important aspects brought by Bentham Obras in the construction management.
\end{abstract}

\section{KEYWORDS}

Construction, Environment, Mobile Application, Web System

\section{INTRODUÇÃO}

Nas últimas décadas, com o surgimento e evolução das Tecnologias de Informação e Comunicação (TICs), vários setores sociais e industriais estão passando por um processo de modernização. Nesse contexto, as TICs vêm desempenhando um importante papel, na medida em que fornecem um conjunto de recursos tecnológicos que podem ser utilizados para o desenvolvimento de aplicações Web e Mobile, as quais podem automatizar e otimizar o gerenciamento de grande volume de dados.

Educação, saúde, segurança, esporte/lazer e infraestrutura são exemplos de áreas que as TICs podem atuar. O trabalho de Pereira e Silva [7] enfatiza que a aplicação dessas tecnologias no meio social pode ser benéfica, gerando bem-estar, crescimento econômico e melhoria na qualidade de vida e serviços.

Além disso, existe a Indústria 4.0, que remete a altas taxas de inovação e integração com novas tecnologias no setor industrial [6].

In: XVIII Workshop de Ferramentas e Aplicações (WFA 2019), Rio de Janeiro, Brasil Anais Estendidos do Simpósio Brasileiro de Sistemas Multimídia e Web (WebMedia). Porto Alegre: Sociedade Brasileira de Computação, 2019.

ISSN 2596-1683

\author{
Reinaldo Braga \\ reinaldo.braga@ifce.edu.br \\ Instituto Federal do Ceará
}

Esse termo surgiu na Alemanha como uma proposta para o desenvolvimento de uma nova ideia de política econômica baseada em estratégias de alta tecnologia. O conceito desse termo é baseado em tecnologias como sistemas ciber-físicos e Internet das coisas (Internet of Things - IoT).

A Confederação Nacional da Indústria $(\mathrm{CNI})^{1}$ afirma que a tecnologia aplicada ao processo produtivo e à gestão dos negócios é um trunfo na busca pela produtividade. Em matéria publicada pela Confederação é apresentado um estudo de caso no qual uma empresa multinacional obteve redução de $40 \%$ em seus custos de manutenção e ganhou até $10 \%$ de eficiência em seu maquinário após aplicar conceitos da Indústria $4.0 \mathrm{em}$ seus processos produtivos [2]. Portanto, vista a importância e os benefícios da Indústria 4.0, quanto mais setores estiverem integrados com o conceito, melhores serão os produtos e serviços ofertados.

A área de Infraestrutura, mais especificamente de Construção Civil, tem um grande impacto sobre diversos setores sociais. Por meio dessa área, todos os anos, são construídas universidades, escolas, hospitais, prédios governamentais, empresas, conjuntos habitacionais, residências, viadutos, dentre outros. Percebe-se, então, que a Construção Civil pode beneficiar diversas áreas como educação, saúde e segurança ao fornecer a infraestrutura adequada para a realização de suas atividades.

Embora seja um setor de grande impacto, a Construção Civil, bem como boa parte do setor industrial brasileiro, ainda está aquém da Indústria $4.0[1,2]$. Processos administrativos e gerenciais como a gestão de obras, materiais, equipamentos, contratos, controle de qualidade e financeiro, muitas vezes são registrados em formulários de papel, o que, além de ser um inconveniente do ponto de vista ambiental, também se mostra desvantajoso do ponto de vista operacional, já que o uso de sistemas informatizados é bem mais eficiente.

Assim, dada a importância da Construção Civil para a sociedade e os benefícios provindos da integração do setor com as TICs e a Indústria 4.0, o presente artigo apresenta o Bentham Obras, um sistema de gestão inteligente para a construção civil. $O$ sistema é dividido em dois módulos: Uma versão $W e b$ voltada para utilização em escritório e um aplicativo Mobile para utilização em campo, como, por exemplo, no canteiro de obras.

\footnotetext{
${ }^{1}$ http://www.portaldaindustria.com.br/cni/
} 
O Bentham Obras dá suporte a diversos processos administrativos e gerenciais, tais como acompanhamento financeiro, controle de execução e cumprimento de contratos, acompanhamento de cronogramas, gestão de aspectos ambientais (ex: licenças ambientais e jazidas) e acompanhamento de não conformidades. Além disso, o Bentham Obras é capaz de gerar inúmeros tipos de relatórios gerenciais e gráficos, exibindo uma série de visões de forma clara e intuitiva, otimizando o processo de tomada de decisão dos diversos atores de uma obra, como engenheiros, empresas contratadas e contratante, supervisores, administradores, técnicos e coordenadores.

Dado seu alto grau de inovação, o desenvolvimento do Bentham Obras foi fomentado por meio de uma parceria com a Empresa Brasileira de Pesquisa e Inovação Industrial (EMBRAPII) ${ }^{2}$. A solução foi desenvolvida pelo Laboratório de Redes de Computadores e Sistemas (LAR) do Instituto Federal de Educação, Ciência e Tecnologia do Ceará (IFCE) a partir da demanda da Quanta Consultoria Ltda, uma empresa com atuação a nível nacional e internacional que opera nas áreas de consultoria, engenharia e planejamento urbano [4].

O Bentham Obras foi implantado na Quanta Consultoria Ltda após alcançar uma versão estável. Como primeira utilização comercial, o sistema foi utilizado para gerir um contrato da empresa licitado pela Secretaria Municipal do Amazonas para a supervisão de obras de construção de 4 escolas, no Projeto de Expansão e Melhoria Educacional da Rede Pública Municipal de Manaus (PROEMEM). O sistema está em operação no contexto desse projeto desde novembro de 2018, atendendo cerca de 18 usuários.

O restante do artigo está organizado da seguinte forma: a Seção 2 apresenta os materiais e métodos utilizados para o desenvolvimento do sistema; na Seção 3 são expostos os resultados e discussões, apresentando as principais funcionalidades do sistema proposto; e, por fim, a Seção 4 apresenta as conclusões.

\section{MATERIAIS E MÉTODOS}

Nesta seção, são descritos os materiais utilizados para a elaboração do Bentham Obras e o conjunto de normas básicas estabelecidas para que a solução computacional fosse desenvolvida de maneira eficiente.

O sistema foi desenvolvido com base na metodologia Scrum, um framework que permite que profissionais tratem e resolvam problemas complexos e adaptativos, enquanto produtiva e criativamente entregam produtos com o mais alto valor possível [8]. O projeto levou 1 ano para ser concluído e foi divido em 12 Sprints.

\subsection{Levantamento de Requisitos}

No levantamento de requisitos foi seguido um processo de interação e reuniões com os stakeholders do sistema (gestores de contratos de obra da Quanta) para descobrir suas necessidades e ter uma compreensão do domínio da aplicação. Por esse processo foi levantado um conjunto não estruturado dos requisitos que, posteriormente, foram organizados em grupos coerentes. Além disso, foi necessário definir a prioridade para os requisitos mais importantes e solucionar os conflitos que ocorreram no seu levantamento. Por último, realizou-se a verificação dos requisitos para descobrir se estavam

\footnotetext{
${ }^{2}$ https://embrapii.org.br
}

completos, consistentes e em concordância com o que os stakeholders desejavam do sistema. Após esse processo, os seguintes módulos foram definidos para a aplicação Web e para o aplicativo Mobile:

(1) Módulo de Planejamento de Programa (Web): Assessora na revisão, nos prazos de entrega e execução de serviços, alocação de recursos humanos, equipamentos e fornecimento de apoio logístico às obras no cronograma. O módulo também controla a programação físico-financeira das obras, verificando sua execução e auxiliando eventuais adequações às necessidades da obra. A plataforma gera relatórios de Estrutura Analítica do Programa (EAP), de cláusulas contratuais e do plano de aquisições para o acompanhamento.

(2) Módulo de Controle de Contratos (Web e Mobile): Auxilia no acompanhamento e execução dos contratos das obras, compreendendo a análise e o parecer de aditivação desses contratos, o controle orçamentário das obras e o apoio no acompanhamento.

(3) Módulo de Iniciar Jornada (Mobile): Auxilia na execução, controle e monitoramento de informações para uso no APP, permitindo que o usuário tenha acesso a tais informações mesmo quando não está conectado à Internet.

(4) Módulo de Controle de Qualidade (Web e Mobile): Analisa os métodos construtivos utilizados e sua adequabilidade à qualidade e prazo das obras. A ISO 9001 é uma norma pertencente à família 9000 e traz os requisitos do Sistema de Gestão de Qualidade para que a empresa possa alcançar resultados em seus processos por meio da eficácia e da qualidade [3]. Assim, o não atendimento a um requisito é definido como uma não conformidade.

(5) Módulo de Meio Ambiente (Web): Auxilia nos processos de gerenciamento ambiental como o controle de licenças ambientais e jazidas, estudos, monitoramento dos impedimentos por fatores ambientais da obra, dentre outros.

\subsection{Arquitetura}

Em paralelo ao levantamento de requisitos, foi realizado um estudo técnico das tecnologias mais adequadas para atender às demandas do sistema nas versões Web e Mobile.

A Figura 1 apresenta a arquitetura do sistema Bentham Obras.

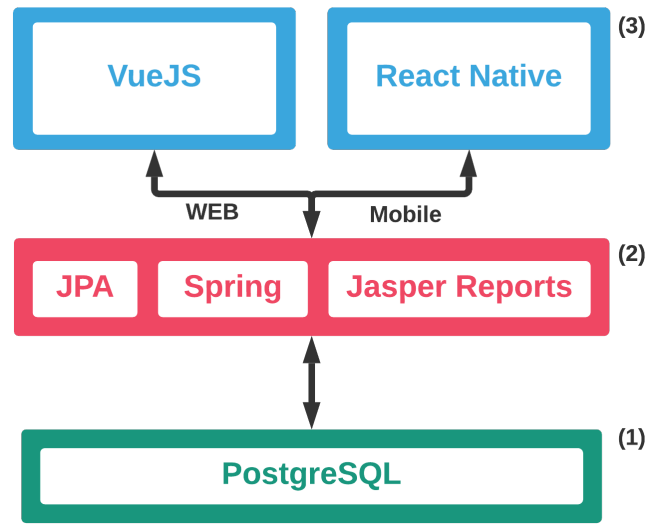

Figure 1: Arquitetura do Bentham Obras. 
(1) Na camada inferior está o Sistema de Gerenciamento de Banco de Dados (SGBD) usado no sistema, o PostgreSQL. Esse SGBD utiliza a Linguagem de Consulta Estruturada (SQL) como interface e, combinada com recursos de armazenamento, é capaz de dimensionar com segurança as cargas de trabalho de dados mais complexas, oferecendo confiabilidade, integridade e escalabilidade de dados.

(2) Na camada central está implementado o Spring, um framework Java para desenvolvimento de sistemas Web e Mobile que utiliza o padrão Model-View-Controller (MVC). O mesmo permite a divisão das funcionalidades de um software em camadas, abstraindo os detalhes de infraestrutura, permitindo ao programador se dedicar às regras de negócio da aplicação. Nessa camada também foi utilizado o fava Persistence API (JPA), que descreve a interface padrão para frameworks de persistência de dados; e o fasper Reports, responsável pela geração de relatórios em formatos PDF e XLS.

(3) Na camada superior está o Front-End, que está dividido em duas partes: uma para navegadores Web e outra para dispositivos Mobile. Na parte Web é utilizado o VuefS, um framework baseado em componentes Web que reúne HTML, CSS e JavaScript, facilitando a adoção e integração com outras bibliotecas e construindo sofisticadas Single-Page Applications. Na aplicação Mobile é utilizado o React Native, um framework que provê componentes que gerenciam o estado interno da plataforma utilizando JavaScript. Assim, foi possível o desenvolvimento de aplicações tanto para dispositivos Android, como iOS.

\section{DESENVOLVIMENTO}

Após o levantamento de requisitos e a definição das tecnologias, iniciou-se o ciclo de desenvolvimento do sistema. Nesta sprint, foram elaborados os diagramas dos fluxos da regra de negócio usando Linguagem de Modelagem Unificada (UML), o modelo de banco de dados, os mockups das interfaces do usuário e os modelos dos relatórios.

A primeira tarefa realizada no desenvolvimento do sistema foi a modelagem do banco de dados, adaptando a escalabilidade da base levando em consideração que o sistema poderia vir a sofrer mudanças futuras. Além disso, o sistema Bentham Obras foi pensado com foco na usabilidade do usuário. Por isso, suas interfaces se apresentam de modo fluido e intuitivo para auxiliar o usuário na sua alimentação e navegação.

Quanto à segurança, foi utilizado o Spring Security, um framework que visa fornecer autenticação e autorização de maneira prática para aplicativos Java ${ }^{3}$. A ferramenta é fácil de configurar e personalizar, o que a torna acessível e adaptável a todo tipo de projeto. Além disso, é muito utilizada e difundida pela comunidade Java através de fóruns de desenvolvedores [5].

Utilizou-se também o fson Web Token (JWT), um padrão de mercado que define como transmitir e armazenar objetos JSON de forma compacta e segura entre diferentes aplicações. Os dados nele contidos podem ser validados a qualquer momento, pois o token é assinado digitalmente. Nesse token são guardados os dados do

\footnotetext{
${ }^{3}$ https://spring.io/projects/spring-security
}

usuário e permissões de acesso ao sistema, evitando, assim, ter que lidar com problemas de sessão expirada.

\subsection{Validação e Testes}

Nesta etapa final, os requisitos e as funcionalidades desenvolvidas passaram por um processo rigoroso de validação de software com a equipe de testes verificando as funcionalidades com dados reais no sistema Web e no aplicativo Mobile para descobrir falhas e avaliar o comportamento das telas do sistema.

Além disso, um grupo de stakeholders da Quanta verificou se o sistema estava funcional e se contemplava o que foi definido no seu escopo. Dessa forma, assegurou-se que todas as funcionalidades foram implementadas corretamente na perspectiva do cliente final.

\subsection{Licença de Software}

O sistema Bentham Obras possui o tipo de licença de software proprietário. A Quanta Consultoria Ltda detém todos os direitos de distribuição, comercialização e uso.

\section{RESULTADOS E DISCUSSÕES}

A partir do que foi exposto, tem-se um sistema Web e um aplicativo Mobile capazes de interagir com dados de obras de construção civil e, a partir disso, gerar relatórios, informações gráficas e textuais suficientes para facilitar o controle de programas e contratos de obras de engenharia civil.

Por questão de espaço, nesta seção são apresentadas apenas algumas das funcionalidades mais relevantes de cada módulo do Bentham Obras, que são aplicadas com dados de teste disponibilizados pela Quanta nas soluções Web e Mobile.

\subsection{Módulo de Planejamento de Programa}

O Módulo de Planejamento de Programa é responsável pelo apoio na gestão, monitoramento e avaliação de um programa; controle, acompanhamento e execução de contratos e apoio técnico geral. Assim, o sistema possui uma visão com base no plano contratual dos contratos e no seu cronograma financeiro.

Através de um conjunto de formulários de cadastro, como o ilustrado na Figura 2, este módulo fornece uma visão geral dos objetivos da empreitada, apoiando o processo de tomada de decisão dos gestores no que concerne aos contratos que ainda não tiveram sua execução iniciada.

\subsection{Módulo de Controle de Contratos}

Conforme apresentado na Seção 2.1, o módulo é responsável por apoiar o acompanhamento administrativo e controle orçamentário dos contratos em execução, além dos custos de cada obra, compreendendo o gerenciamento da quantidade e preços dos insumos utilizados em cada contrato. O sistema pode, por exemplo, informar o percentual de insumos já utilizados em cada etapa da execução do contrato e, através disso, é possível saber quanto ainda se pode gastar, de acordo com o valor orçado e acordado no contrato.

Na Figura 3 é apresentado um exemplo de Gráfico $S$, que mostra a evolução da execução de um contrato. Nota-se que o histograma mostra o valor gasto em cada mês no canteiro de obras. Na parte inferior da tela o usuário pode acompanhar o valor que foi estipulado para ser gasto até a etapa atual, mas que ainda não foi utilizado 


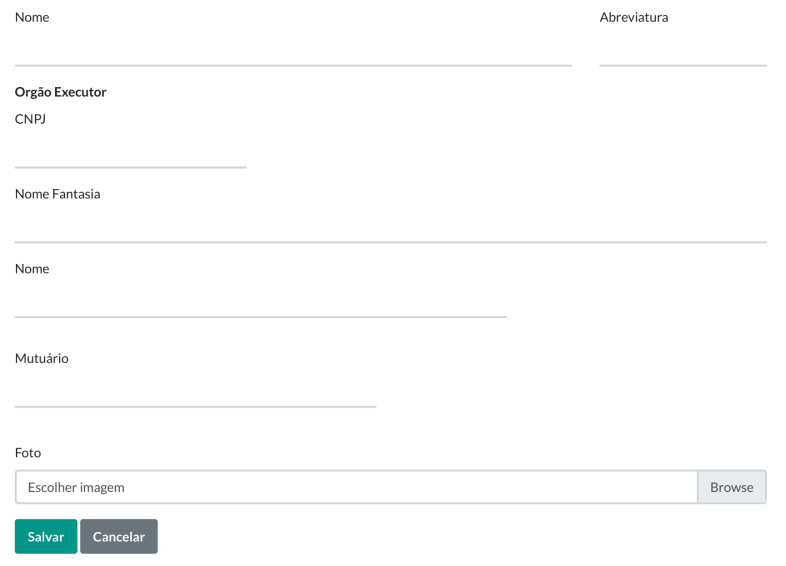

Figure 2: Tela de Cadastro de Programa do Bentham Obras.

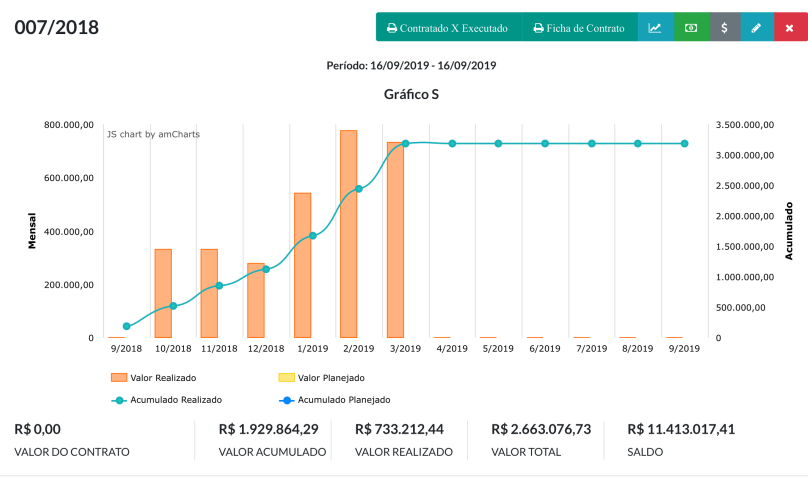

Figure 3: Tela de Gráfico $S$ do Bentham Obras.

(VALOR ACUMULADO); o valor que foi de fato utilizado até o momento (VALOR REALIZADO); a soma desses dois valores (VALOR TOTAL) e, além disso, quanto ainda falta para ser utilizado nas demais etapas (SALDO).

\subsection{Módulo de Iniciar Jornada}

O aplicativo Mobile do Bentham Obras tem uma particularidade interessante: parte de suas funcionalidades pode ser utilizada em modo offline.

Depois que o usuário realiza login no aplicativo, ele é direcionado para a tela Iniciar fornada, como ilustra da tela da Figura 4(a). Ao clicar no botão Iniciar Jornada, o usuário dá início a uma nova jornada de trabalho, definindo o ponto de partida para uma nova etapa da obra. Esta é uma das poucas operações que requer conexão com a Internet. O usuário deve especificar o programa e o contrato que deseja trabalhar. Em seguida, ele pode visualizar, editar e excluir diários e notificações dentro de um determinado período de dias, meses ou anos.

Iniciada uma fornada, todas as demais operações feitas no aplicativo são salvas offline, na memória local do dispositivo. Somente quando o usuário finaliza a jornada e tem acesso à Internet, é que essas informações são enviadas para o banco de dados online.

Assim, o aplicativo Mobile pode ser utilizado no canteiro de obras, onde muitas vezes não há acesso à Internet, dando maior mobilidade ao sistema.

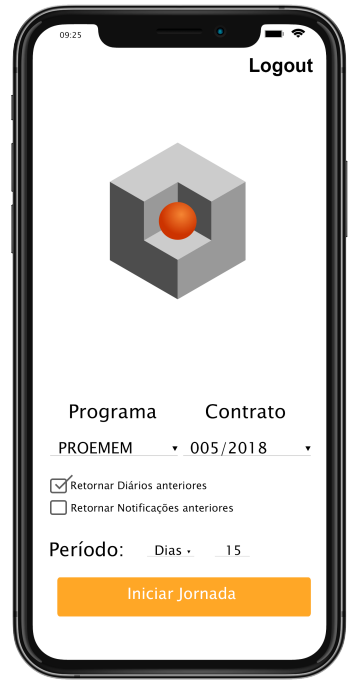

(a) Iniciar fornada.

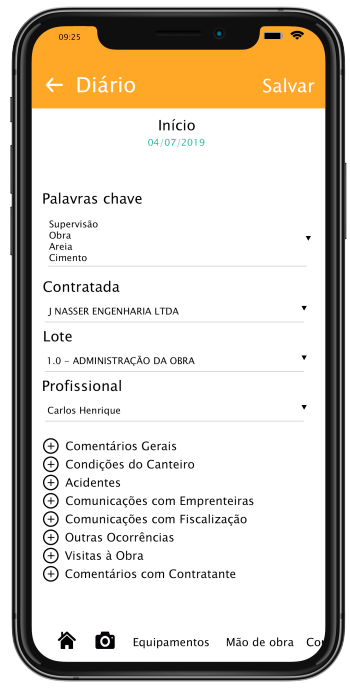

(b) Diário de Obras.
Figure 4: Telas do aplicativo Mobile do Bentham Obras.

\subsection{Módulo de Diário de Obras}

Após o inicio de uma fornada, o usuário também tem a opção de adicionar um Diário de Obras, conforme ilustra a tela da Figura 4(b). Nele é possível definir uma série de aspectos relativos à execução do contrato e da obra, tais como: cronogramas de entrega e montagem de equipamentos; programação de cronogramas físico-financeiros; rotinas de revisão e atualização de programações; emissão de pareceres; alocação de recursos humanos, equipamentos de informática e escritório; mecanismos de apoio logístico às obras, dentre outros. Essa gama de informações provê aos supervisores uma maior facilidade no processo de monitoramento e controle.

\subsection{Módulo de Controle de Qualidade}

O Módulo de Controle de Qualidade permite ao usuário notificar uma não conformidade, ou seja, um não cumprimento dos contratos e dos padrões de qualidade. É possível realizar a análise dos métodos utilizados e sua adequabilidade à qualidade e prazo das obras. O módulo oferece suporte à assessoria na supervisão das obras e serviços do programa de acordo com os contratos. A Figura 5 apresenta a tela de cadastro de controle de qualidade.

Após a detecção de uma não conformidade, em um processo de auditoria ou no dia a dia da instituição, é elaborado um relatório de auditoria interna ou externa com os envolvidos para o registro da ação e análise da causa. 
O controle de qualidade segue um fluxo de estados para fazer a análise da causa, chegar à problemática e desenvolver a ação preventiva/corretiva, tudo isso com prazos estabelecidos em um plano de ação com o responsável.

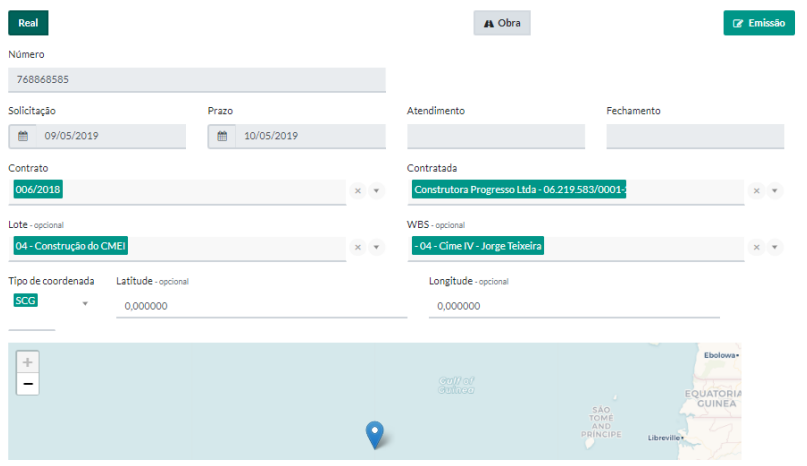

Figure 5: Tela de Cadastro de Controle de Qualidade do Bentham Obras.

\subsection{Módulo de Meio Ambiente}

Há também o Módulo de Meio Ambiente do Bentham Obras, fundamental para consolidar o desenvolvimento sustentável e regular a execução de atividades potencialmente prejudiciais a áreas ambientais. Nesse módulo são cadastradas as Licenças Ambientais e fazidas, que fornecem dados sobre a regularidade e viabilidade ambiental da obra, além de estabelecer limites de atuação.

A Figura 6 ilustra a tela de cadastro de licença ambiental. As Licenças Ambientais são cadastradas de acordo com os serviços que serão executados na obra. Ao todo, há três tipos de licença: licença da obra; licença de supressão vegetal; e licença de captura, coleta e transporte de fauna. A licença em si só pode ser obtida através de órgãos governamentais. Somente a partir daí é anexada ao sistema para um controle mais dinâmico de seus aspectos.

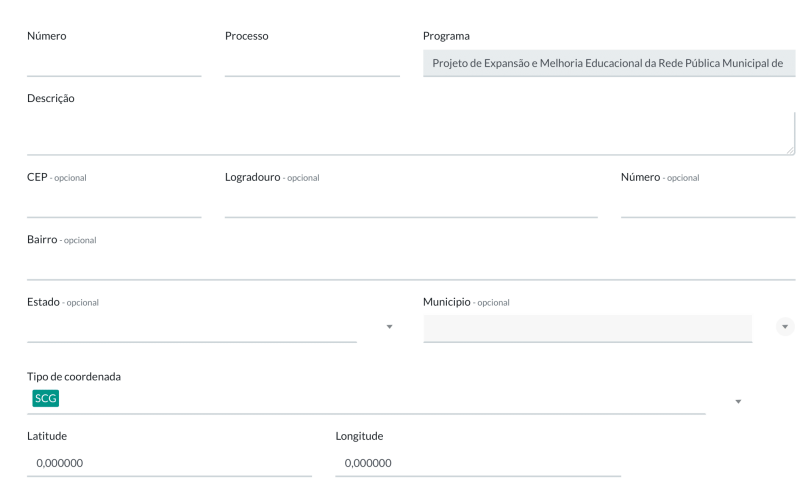

Figure 6: Tela de Cadastro de Licença Ambiental do Bentham Obras.

Já as fazidas são cadastradas de acordo com materiais naturais, em geral minérios, que podem ser explorados, como brita, areia, calcário, dentre outros. No sistema deve-se informar o código identificador dos documentos oficiais de cada jazida cuja exploração foi liberada pelo governo.

\section{CONCLUSÕES}

O Bentham Obras, através das soluções Web e Mobile apresentadas, pode levar os processos gerenciais de obras a uma série de melhorias, que vão desde um melhor controle sobre os gastos com mão de obra e materiais, até um controle centralizado sobre licenças ambientais e qualidade de serviços.

Através do Diário de Obras, por exemplo, o sistema oferece um amplo controle sobre as atividades realizadas diariamente no canteiro. A cada dia são fornecidos dados informando tudo o que foi gasto e com o que foi gasto; então esses dados são agrupados e convertidos em informações, as quais são apresentadas em gráficos de forma clara e intuitiva.

Em suma, é notável que o Bentham Obras agrega valor nos processos de planejamento, execução e controle de obras de construção civil, permitindo uma evolução constante na melhoria de processos e, inclusive, na qualificação dos profissionais envolvidos.

\section{REFERENCES}

[1] Confederação Nacional de Indústria (CNI). 2018. Mapa Estratégico da Indústria 2018-2022. Brasília, DF.

[2] Confederação Nacional de Indústria (CNI). 2018. Mapa Estratégico da Indústria 2018-2022, Melhora na produtividade brasileira depende de ação das empresas. Retrieved 19 de Julho, 2019 from http://www.portaldaindustria. com.br/cni/canais/mapa-estrategico-da-industria/reportagem-especial/ capitulo-12-melhora-na-produtividade-brasileira-depende-de-acao-das-empresas/

[3] Portal ISO. 2019. Retrieved 18 de Julho, 2019 from https://iso9001.portaliso.com/ normas-iso-9000/

[4] Quanta Consultoria Ltda. [n.d.]. Retrieved 20 de Julho, 2019 from https://www. quantaconsultoria.com

[5] N. Meng, S. Nagy, D. Yao, W. Zhuang, and G. Arango-Argoty. 2018. Secure Coding Practices in Java: Challenges and Vulnerabilities. In IEEE/ACM International Conference on Software Engineering (ICSE). 372-383.

[6] T. Oesterreich and F. Teuteberg. 2016. Understanding the implications of digitisation and automation in the context of Industry 4.0: A triangulation approach and elements of a research agenda for the construction industry. Computer in industry 83,10 (2016), $121-139$.

[7] D. Pereira and G. Silva. 2010. As Tecnologias de Informação e Comunicação (TICs) como aliadas para o desenvolvimento. Cadernos de Ciências Sociais Aplicadas Vitória da Conquista (BA) 10 (2010), 151 - 174.

[8] K. Schwaber and J. Sutherland. 2013. Guia do Scrum ${ }^{\mathrm{TM}}$. In Um guia definitivo para o Scrum: As regras do jogo. Retrieved 20 de Julho, 2019 from https://www. scrumguides.org/docs/scrumguide/v1/Scrum-Guide-Portuguese-BR.pdf 